\title{
Intra-Datacenter Interconnects with a Serialized Silicon Optical Frequency Comb
} Modulator

Kong, Deming; Xin, Haiyun; Kim, Kwangwoong; Liu, Yong; Oxenløwe, Leif Katsuo; Dong, Po; Hu, Hao

Published in:

Journal of Lightwave Technology

Link to article, DOI:

10.1109/JLT.2020.2996410

Publication date:

2020

Document Version

Peer reviewed version

Link back to DTU Orbit

Citation (APA):

Kong, D., Xin, H., Kim, K., Liu, Y., Oxenløwe, L. K., Dong, P., \& Hu, H. (2020). Intra-Datacenter Interconnects with a Serialized Silicon Optical Frequency Comb Modulator. Journal of Lightwave Technology, 38(17), 4677 4682. https://doi.org/10.1109/JLT.2020.2996410

\section{General rights}

Copyright and moral rights for the publications made accessible in the public portal are retained by the authors and/or other copyright owners and it is a condition of accessing publications that users recognise and abide by the legal requirements associated with these rights.

- Users may download and print one copy of any publication from the public portal for the purpose of private study or research.

- You may not further distribute the material or use it for any profit-making activity or commercial gain

- You may freely distribute the URL identifying the publication in the public portal 


\title{
Intra-Datacenter Interconnects with a Serialized Silicon Optical Frequency Comb Modulator
}

\author{
Deming Kong, Member, IEEE, Member, OSA, Haiyun Xin, Kwangwoong Kim, Yong Liu, Student Member, OSA, \\ Leif Katsuo Oxenløwe, Member, IEEE, Fellow, OSA, Po Dong, Fellow, IEEE, Fellow, OSA, \\ and $\mathrm{Hao} \mathrm{Hu}$, Member, IEEE, Member, OSA
}

\begin{abstract}
Powerful warehouse-scale datacenters form the fabric of cloud computing. Efficient computing requires intradatacenter interconnects with large capacity, low energy consumption, and high scalability. These unique goals present brand new scientific and technological challenges for short-reach optical communications. Silicon photonics ( $\mathrm{SiP}$ ) modulators are promising for intra-datacenter interconnects for its low energy consumption, with the superiority of low cost, small footprint, and complementary metal-oxide-semiconductor (CMOS) compatibility. While currently, the main research focus is to increase the modulation speed of a single $\mathrm{SiP}$ modulator for a large interconnect capacity, parallel schemes exhibit better scalability. In this paper, we propose intra-datacenter interconnects using dense wavelength-division multiplexing (DWDM) techniques with a single SiP optical frequency comb modulator (OFCM) to achieve potential large capacity and high scalability. The SiP-OFCM consists of serially cascaded microring modulators (MRMs). The MRM based SiP-OFCM is intrinsically compatible with DWDM, performing simultaneously data modulation for multiple optical carriers, DWDM demultiplexing and multiplexing functionalities. We demonstrate an interconnect line rate of $400 \mathrm{Gbit} / \mathrm{s}$ with a PAM 4 modulation format using the SiP-OFCM. All four DWDM channels exhibit bit-error ratios below the $33 \%$ hard-decision forward-error correction threshold after $2-\mathbf{k m}$ single-mode fiber transmission.
\end{abstract}

Index Terms-Intra-datacenter interconnects, optical frequency comb, integrated photonics, silicon photonics.

\section{INTRODUCTION}

$\mathbf{E}$ XTREMELY powerful warehouse-scale data centers are the foundation of Internet applications such as cloud computing. Computing tasks within these data centers rely on the sharing of computing resources to achieve efficiency, coherence, and economies of scale. The intra-datacenter interconnects of servers, racks, and switches require not only large capacity but, most importantly, high scalability and low energy consumption. These requirements propose new scientific and

Manuscript received January 13, 2020. This work is supported by a research grant (15401) of the Young Investigator Program (2MAC) from the VILLUM FONDEN, and DNRF Research Centre of Excellence, SPOC (ref. DNRF123). Corresponding authors: Deming Kong and $\mathrm{Hao} \mathrm{Hu}$

D. Kong, Y. Liu, L.K. Oxenløwe, and H. Hu are with the Department of Photonics Engineering, Technical University of Denmark, Kongens Lyngby 2800, Denmark (e-mail: dmkon@fotonik.dtu.dk; yonli@fotonik.dtu.dk; lkox@fotonik.dtu.dk; huhao@fotonik.dtu.dk).

K. Kim and P. Dong are with Nokia Bell Labs, 791 Holmdel Road, Holmdel, New Jersey 07733, USA (e-mail: kw.kim@nokia-bell-labs.com; po.dong@nokia-bell-labs.com).

H. Xin is with State Key Laboratory of Advanced Optical Communication Systems and Networks, Shanghai Jiao Tong University, Shanghai 200240, China. technological challenges for short-reach optical communications. Silicon photonics (SiP) modulators have become an attractive solution to intra-datacenter interconnects due to small footprint, low energy consumption, and complementary metal-oxide-semiconductor (CMOS) compatibility. Many SiP devices have been demonstrated for optical transceivers, including modulators [1], spectral filters [2], [3], and photodetectors [4]. Among these components, the SiP modulator is the key to improve the overall capacity. High-speed SiP modulators, including electroabsorption modulators (EAM) [5]-[7], Mach-Zehnder modulators (MZM) [8]-[12], and microring modulators (MRM) [13]-[16] have shown great performance and potential for high-density and low-power monolithically integrated transceivers for short-reach applications. EAMs by epitaxial growth of $\mathrm{Ge}$ on $\mathrm{Si}$ are limited to the working wavelength at the $\mathrm{L}$ band and higher $\mathrm{C}$ band. SiP MZMs have shown advantages of large electro-optical bandwidth and thermal stability, with demonstrated capacity of over $100 \mathrm{~Gb} / \mathrm{s}$ by discrete multitone modulation [12], and pulse-amplitude modulation (PAM) signaling [8]-[10]. Proposals to achieve a capacity to $400 \mathrm{~Gb} / \mathrm{s}$ or even higher have concentrated on either to increase the single-lane bitrate [17] or the number of parallel lanes [9]. Increasing the single-lane bitrate requires a higher baud rate and more modulation levels, which can be very challenging in terms of available signal-to-noise ratio (SNR) and scaling of power efficiency [18]. On the other hand, parallel solutions, including parallel single-mode (PSM) [9] and coarse wavelength-division multiplexing (CWDM) [19], could offer good scalability and large capacity at lower SNR levels. For further increase of the capacity and scalability, dense wavelength-division multiplexing (DWDM) integrated into a single transmitter may be a solution. However, it is very challenging to utilize DWDM for intra-datacenter interconnects. Firstly, DWDM usually requires multiple light sources and active wavelength controls, which consumes extra energy and increases the cost. Secondly, it requires multiplexers and demultiplexers with sharp roll-off and long-term wavelength stability, which will further increase the complexity of the system. Thirdly, it is challenging to integrate many modulators (such as SiP MZMs) and spectral filters (such as SiP arrayed waveguide gratings) on a small footprint chip.

In this paper, we propose a DWDM based intra-datacenter interconnect scheme using a single $\mathrm{SiP}$ optical frequency comb modulator (OFCM), and an electro-optical frequency comb based on a single distributed-feedback (DFB) laser. The OFCM consists of serially cascaded SiP microring mod- 


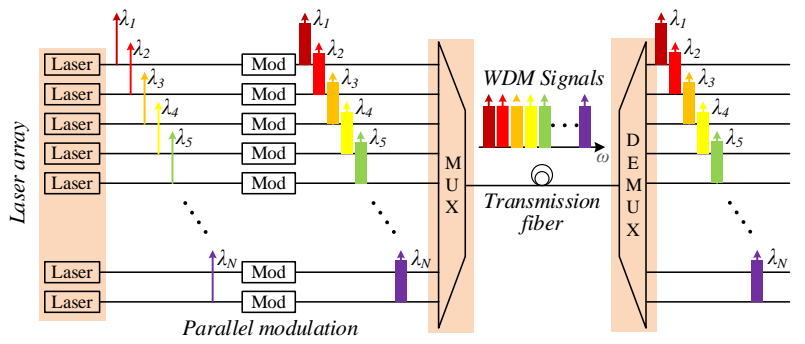

(a)

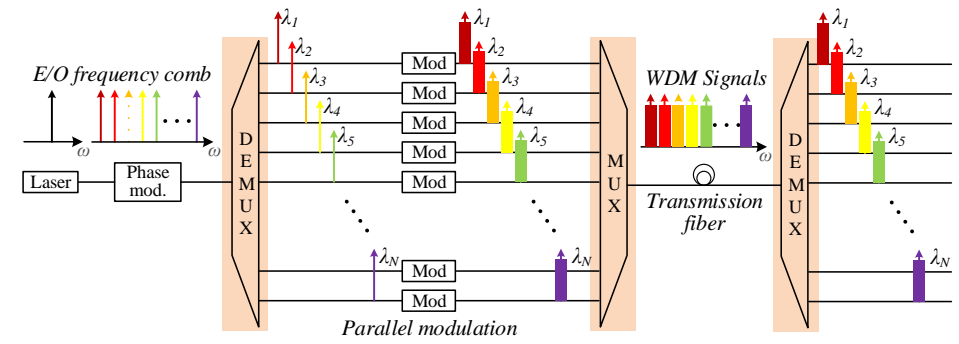

(b)

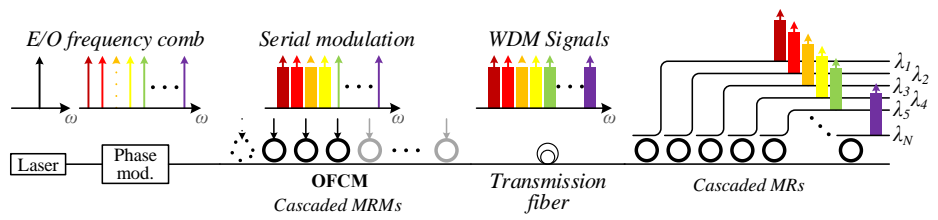

(c)

Fig. 1. Schemes of intra-datacenter interconnect with DWDM based on (a) laser arrays, multiplexers (MUX), and demultiplexers (DEMUX); (b) Electro-optical (E/O) frequency comb, MUX, and DEMUX; and (c) the proposed E/O frequency comb and OFCM.

ulators (MRMs). The MRMs feature several unique advantages that could potentially solve the challenges to apply DWDM in intra-datacenter interconnects. First and foremost, the strong wavelength-selectivity makes MRMs a perfect match with DWDM source, such as energy-efficient electrooptical frequency comb. The integrated MRMs can simultaneously achieve frequency comb demultiplexing, data modulation, and DWDM multiplexing [16]. Second, MRMs have a small footprint and ultra-low power consumption [15], [20]. Combined with the wavelength-selectivity, it is promising for a densely integrated transmitter to achieve high data throughput. Third, the wavelength tunability and cascadability make MRMs highly flexible for elastic optical interconnects. For the frequency comb source, the electro-optical frequency comb requires only a single continuous-wave laser with active wavelength control, which could potentially reduce the energy consumption to tens of milliwatts if integrated on-chip [21]. Catering together these two techniques, we demonstrate the generation of up to $4 \times 50$ Gbaud DWDM-PAM4 signals for intra-datacenter interconnection. Bit-error ratio (BER) performance below $33 \%$ hard-decision forward error correction (HDFEC) threshold is achieved for all four DWDM channels after $2-\mathrm{km}$ transmission with a net rate of $300.75 \mathrm{~Gb} / \mathrm{s}$. At $40 \mathrm{Gbaud}$ modulation, a net rate of $299.06 \mathrm{~Gb} / \mathrm{s}$ is achieved, assuming $7 \%$ HD-FEC threshold. To the best of our knowledge, this is the first DWDM transmission demonstration using a single $\mathrm{SiP}$ integrated circuit with an electro-optical frequency comb source, paving the way for a future single-chip solution with heterogeneous silicon/III-V technology.

The following of the paper structures as follows. Section II gives the principle of the proposed intra-datacenter interconnect system. We show that the proposed OFCM based system simplifies the overall complexity by eliminating the DWDM multiplexer and demultiplexer. Compared with the laser-array based DWDM system, the OFCM based system could also decrease the overall energy consumption. We also briefly introduce the design, fabrication, and packaging of the OFCM. Section III describes the detailed setup of our experiment. Section IV shows the results of the experiment. We also discuss some of the limiting factors of the current packaging design. Section V gives some conclusions.

\section{PRINCIPLE}

\section{AND THE SIP OPTICAL FREQUENCY COMB MODULATOR}

Figure 1(a) shows the scheme of a conventional DWDM system for long-haul transmission, where spectral efficiency is the utmost important factor. This system uses an array of lasers as DWDM sources. Each of the wavelengths is modulated independently by parallel modulators. All the DWDM channels are then multiplexed together by a DWD multiplexer. At the receiver side, the DWDM channels are then demultiplexed by the demultiplexer before detection. This DWDM system may encounter several issues for intra-datacenter interconnects. The laser array requires multiple active wavelength controls, which are energy consuming and costly. The multiplexer and demultiplexer should have long-term wavelength stability and sharp roll-off factors to precisely match the DWDM channels. Optical frequency comb can be utilized as the DWDM sources to solve the energy consumption issue of the laser array. Figure 1(b) shows an improved scheme of a DWDM system, where an electro-optical frequency comb has replaced the laser array. The electro-optical frequency comb has an advantage of high energy efficiency compared with the optical frequency comb generated by utilizing the nonlinear Kerr effect based on highly nonlinear mediums. However, the optical frequency comb must be line-by-line demultiplexed by another DWDM demultiplexer before modulation.

Figure 1(c) shows the proposed solution to the above issues. Instead of parallel modulators, we propose to use an OFCM for all the DWDM channels. The OFCM consists of a serially cascaded array of MRMs, which can be integrated on a single transmitter chip. This scheme comes with some advantages compared with the schemes shown in Fig. 1(a) and Fig. 1(b). The MRM is highly wavelength-selective. Each MRM can register to a single frequency comb line for modulation. The other frequency comb lines will travel along through the bus 


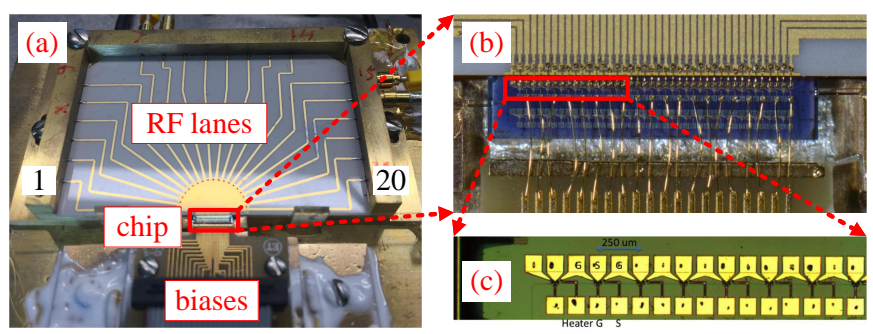

Fig. 2. The silicon OFCM with 20 cascaded MRMs: (a) the packaged device; (b) a zoom-in view of the chip; (c) optical microscopy of the cascaded MRMs.

waveguide until all comb lines are modulated at the output of the OFCM. Therefore, no extra DWDM multiplexer nor demultiplexer is needed. At the receiver side, the serially cascaded microrings can also be used as DWDM demultiplexers. The OFCM can also provide elastic wavelength allocation of the DWDM channels by tuning the working wavelength of each MRM. Combined with the electro-optical frequency comb, this interconnect scheme offers high density, strong scalability, and low energy consumption.

We note that similar chip architectures has been utilized for various applications such as arbitrary waveform generation [22], enhancing filter shape [23], optical switching [24], optical computing [25], optically-connected memory system [26], and optical sensing [27]. This cascaded architecture of MRMs has been conceptually proposed for WDM sources [28]. It has been demonstrated with two concurrently modulated channels with NRZ-shaped signals in the O band [29], [30]. In this paper, we propose to use this cascaded-MRM architecture as an OFCM together with an electro-optical frequency comb, at the conventional $\mathrm{C}$ band. With 20 integrated MRMs in the packaged OFCM, we demonstrate up to $4 \times 50$ Gbaud DWDM-PAM4 transmission over 2-km standard single-mode fiber, with a DWDM channel spacing of $80 \mathrm{GHz}$. The number of DWDM channels is rather limited by our available electrical signal source, than the number of available MRMs.

We designed the OFCM with 20 cascaded MRMs. The radius of the microring is $7.5 \mu \mathrm{m}$, and the spacing between adjacent MRMs is $250 \mu \mathrm{m}$. Each MRM has a microheater sitting on top of it to adjust the resonant wavelengths. The footprint of the microring resonators and the optical bus waveguide is approximately $0.08 \mathrm{~mm}^{2}$. The overall footprint of the OFCM is limited by the electrical devices such as electrical wires, DC and RF bond-pads, and external printed circuit board (PCB) board and drivers. On the contrary, other SiP transmitter schemes utilizing DWDM selective devices such as arrayed waveguide grating (AWG) [2] and MZMs [31](even with an ultra-compact design) are limited by the footprint of the optical devices. The high-speed modulation of our MRMs is based on a reverse-biased P-N junction in the middle of the microring waveguide [15], [16]. The PIC was fabricated on an 8-inch silicon-on-insulator wafer with a top silicon thickness of $220 \mathrm{~nm}$. Figure 2(a)-(c) shows the packaged device, the chip, and microscopy of the cascaded MRMs. We packaged the chip with two fibers, a PCB with 20 $\mathrm{RF}$ transmission lines to drive the modulators, and a second PCB with DC lines to control the heaters. The packaged
MRMs have an estimated bandwidth of around $15 \mathrm{GHz}$, from our previously measured devices with a similar design.

\section{EXPERIMENTAL SETUPS}

We use the packaged OFCM for a DWDM based intradatacenter interconnect experiment. Figure 3 shows the experimental setup. The electro-optical comb originates from a DFB laser working at $1551.88 \mathrm{~nm}$ with an output optical power of $13 \mathrm{dBm}$. The DFB laser is phase-modulated at 40 $\mathrm{GHz}$ through a phase modulator (PM). After amplification through an EDFA (EDFA 1), the generated $40-\mathrm{GHz}$ spaced optical frequency comb is line-by-line filtered and equalized by a wavelength-selective switch (WSS) to a flat-top, 80$\mathrm{GHz}$ spaced optical frequency comb with four comb lines located at $1550.91 \mathrm{~nm}, 1551.55 \mathrm{~nm}, 1552.19 \mathrm{~nm}$, and 1552.83 $\mathrm{nm}$, respectively. The four comb lines are used as DWDM sources and launched into the OFCM. In this experiment, we used MRM 7, 8, 15, and 16 simultaneously to implement the 4-channel DWDM transmitter. The absolute wavelength registration of the MRMs is challenging due to fabrication deviations and ambient temperature changes. The resonance frequencies of the MRMs are tuned by the integrated heaters to match the optical frequency comb lines. The tuning of the working wavelength of the MRMs is around $2 \mathrm{~V} / \mathrm{nm}$. The total consumed power from the heaters on the MRMs is around $120 \mathrm{~mW}$. We note that the resonance frequencies can be stabilized and locked using a single monitoring signal for a transmitter comprising many rings [32], [33]. A WSS is used in this proof-of-concept experiment to equalize the optical frequency comb, which might not be preferred in the intradatacenter interconnect. However, the flat optical frequency comb generation can be replaced by other electro-optical frequency comb generation schemes, which do not need the costly WSS [34]-[36]. The electro-optical comb source can also potentially be integrated on-chip [37].

The four electrical PAM-4 signals are generated using an arbitrary waveform generator (AWG) with a $65 \mathrm{GSa} / \mathrm{s}$ sampling rate from a pseudo-random binary sequence with a length of $2^{15}-1$. A digital root-raised-cosine (RRC) filter with 501 taps and a roll-off factor of 0.01 is applied for pulse shaping. Four broadband electrical amplifiers and four electrical bias tees are used to amplify the 4-channel electrical PAM-4 signals to a peak-to-peak voltage around 5 volts with non-negative amplitudes and drive the MRMs through the packaged RF connectors. The MRMs are thermally tuned to match the wavelengths of the frequency comb using DC lines wired with the heaters. The total optical input power to the transmitter is $4 \mathrm{dBm}$. The optical insertion loss for the whole device is $19 \mathrm{~dB}$, mainly due to the misalignment of the lensed fiber and the chip during the packaging process. The output of the transmitter is amplified by an optical preamplifier (EDFA 2) to $13 \mathrm{dBm}$ and filtered through an optical bandpass filter (OBPF 1). The generated 4-channel DWDM PAM-4 signals are transmitted over 2-km single-mode fiber (SMF) in order to evaluate the transmission performance.

We use a preamplified optical receiver to evaluate the BER performance of the interconnect system. The receiver consists 


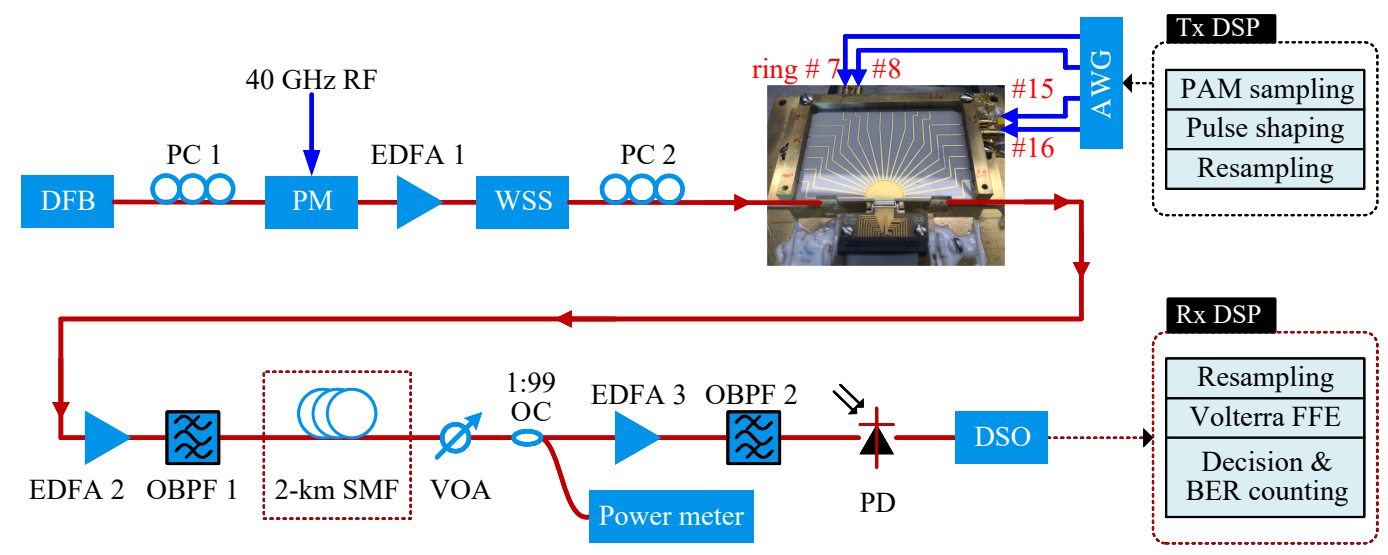

Fig. 3. Experimental setup. (PC: polarization controller; EDFA: erbium-doped fiber amplifier)

of a variable optical attenuator (VOA), a 1:99 optical coupler (OC) with a power meter, an optical preamplifier (EDFA 3), OBPF 2, and a 70-GHz bandwidth photodiode (PD). The VOA is used to adjust the received optical power, which is recorded by the power meter. All measurements are taken with all DWDM channels active at the same time. The received electrical signal is sampled by a digital storage oscilloscope (DSO) with an analog bandwidth of $63 \mathrm{GHz}$ and a sampling rate of $160 \mathrm{GSa} / \mathrm{s}$. Two 4-million-sample records for each received power of each DWDM channel are used for the offline digital signal processing (DSP), including resampling, Volterra-based feedforward equalization (Volterra FFE), and decision and BER count.

We choose the nonlinear Volterra equalizer in this experiment to combat both linear and nonlinear impairments from the transmitter. The main contributing factors to the nonlinear response of the transmitter are limited bandwidth of the MRMs, the insufficient modulation depth due to the high loss of the RF microstrip lines from the packaging, and the nonlinear response of the electrical drivers that worked in the nonlinear region for an increased modulation depth and SNR. These nonlinear issues can, however, be resolved by reducing the modulation speed to match the bandwidth of the MRMs and RF devices. The coefficients of the Volterra feedforward equalizer (FFE) are obtained through training based on the least mean square (LMS) algorithm. The equalizer adopted in back-to-back and 2-km cases are with the same structure, i.e., a general Volterra-based FFE [38]. However, the coefficients are independently obtained according to the received signals in back-to-back and after transmission. The equalizer lengths for the first, second, and third kernels of the Volterra FFE are 101, 29 , and 9 , respectively. To reduce computation complexity, we adopt the simplified method proposed in [39], where kernels with large interval product terms are truncated since they have negligible contribution to signal distortions. For the second and third kernels, kernels with an interval larger than 3 are truncated. This results in a reduced number of kernels from 435 to 110 , and from 165 to 70 , for the second and third kernels, respectively.
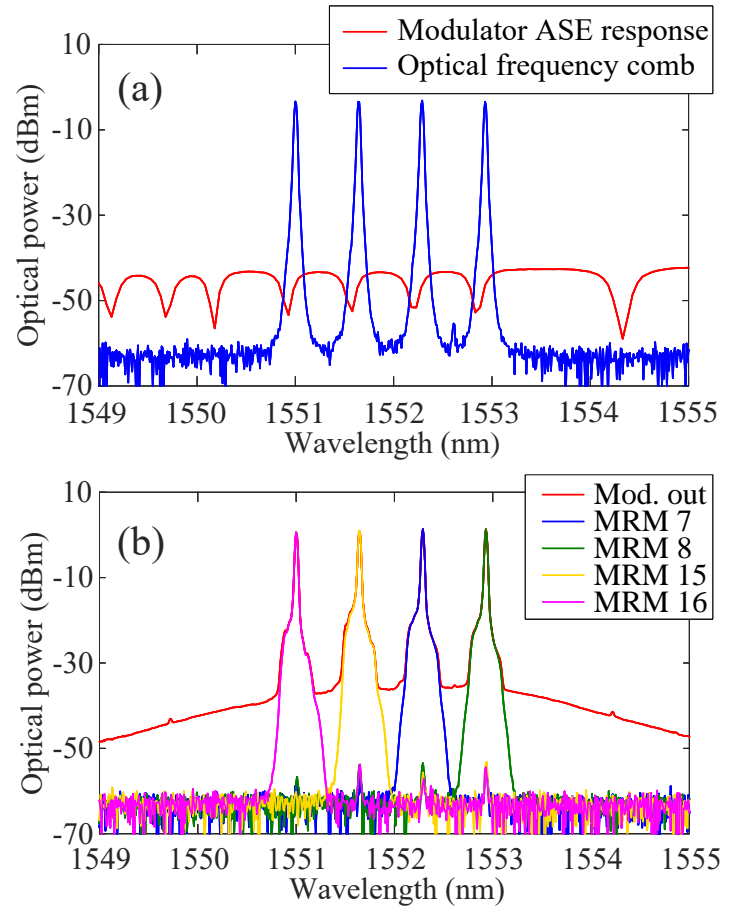

Fig. 4. Optical spectra of (a) the ASE response of the integrated modulator and the optical frequency comb; (b) the output of the modulator and the outputs from each MRM. Resolution bandwidth: $0.1 \mathrm{~nm}$.

\section{RESUlTS AND DISCUSSIONS}

Figure 4(a) shows the optical spectra out of the optical frequency comb modulator with the input of ASE noise, as well as the equalized $80-\mathrm{GHz}$ spaced optical frequency comb source. The power variation of the equalized comb lines is within $1 \mathrm{~dB}$. Figure 4(b) gives the optical spectra of the modulated PAM 4 signals from each MRM, as well as the DWDM signal from the OFCM. The large residual carrier in the optical spectrum is due to limited modulation depth, as the high-frequency electrical components of the driven signals exhibit extra loss due to bandwidth limitation. We choose a channel spacing of $80 \mathrm{GHz}$ to mitigate the intermodulation crosstalk from cascaded MRMs well-below $1 \mathrm{~dB}$ [40]. Yet, as suggested in [40], due to the deterministic nature of the 
intermodulation crosstalk, DSP algorithms such as MIMO can be used to mitigate the crosstalk and achieve a denser DWDM channel spacing.

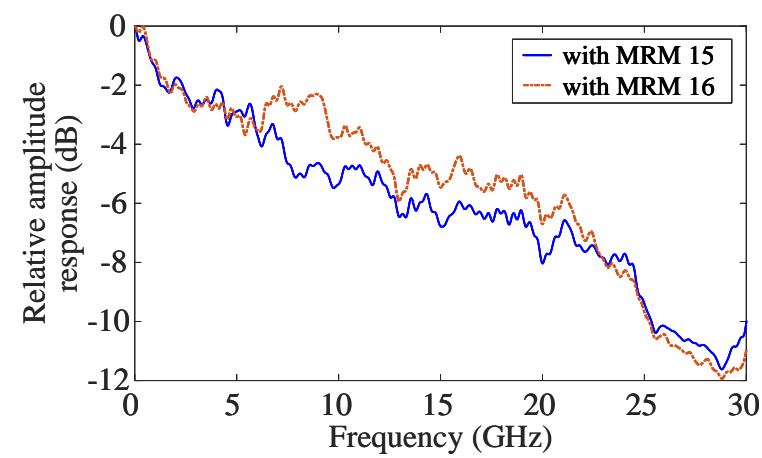

Fig. 5. The measured overall amplitude response of the system with MRM 15 and MRM 16.

Figure 5 is the measured overall amplitude response of the interconnect system with MRM 15 and MRM 16. The amplitude-frequency response includes the AWG, the electrical driver, the MRM, the PD, and the DSO. We can see a more than $10 \mathrm{~dB}$ amplitude drop within the $25-\mathrm{GHz}$ signal bandwidth. This bandwidth limitation mainly comes from the MRM and puts an upper limit for the achievable data rate. The bandwidth limit is very challenging for linear equalization. Thus we use nonlinear Volterra equalizer in this demonstration. We expect to achieve a higher data rate with a simplified linear equalization if the bandwidth of the MRM can be increased. The difference of amplitude-frequency responses for MRM 15 and 16 explains the difference in the BER performance.

Figure 6 (a) and (c) give the BER performance of the $4 \times 40$ Gbaud DWDM-PAM4 signals using LMS linear equalizer in the back-to-back case and after 2-km transmission, respectively. The signals show a BER performance below the $33 \%$ HD-FEC threshold $\left(\mathrm{BER}=2.12 \times 10^{-2}\right.$, assuming staircase codes [41]). Figure 6(b) and (d) give the BER performance of the $4 \times 40$ Gbaud DWDM-PAM4 signals using Volterra FFE, before and after transmission. Compared with Fig. 6(a) and (c), we can see a performance gain by using the Volterra FFE, which brings down the BER floor below 7\% HD-FEC threshold $\left(\mathrm{BER}=3.8 \times 10^{-3}\right)$. For the DWDM channel from MRM 16, there is a $5-\mathrm{dB}$ improvement in terms of receiver sensitivity. The insets of Fig. 6(a)-(d) show the corresponding eye diagrams from the DWDM channel with MRM 16. We can see a larger eye-opening with Volterra FFE than the LMS linear equalizer. Our system is mainly limited by the bandwidth of the MRMs, as well as other nonlinear impairments such as the electrical drivers working at saturation for an adequate voltage swing. We can conclude that the Volterra FFE does help to mitigate these nonlinear impairments and allows an overall better performance. The achieved net rate for the $4 \times 40$ Gbaud DWDM-PAM4 signals is calculated to be $299.06 \mathrm{~Gb} / \mathrm{s}$.

Figure $6(\mathrm{e})$ and (f) are the BER performance of the $4 \times 50$ Gbaud DWDM-PAM4 signals in back-to-back and after 2$\mathrm{km}$ transmission, using Volterra FFE. The BER performance is below the $33 \%$ HD-FEC threshold. A net rate of 300.75 $\mathrm{Gb} / \mathrm{s}$ is achieved. Although the spectral efficiency for intensity-

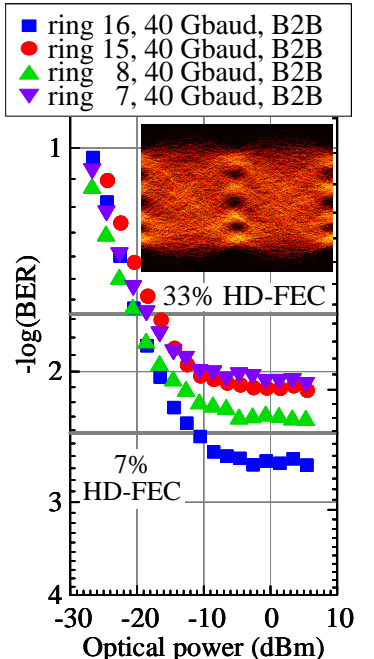

(a)

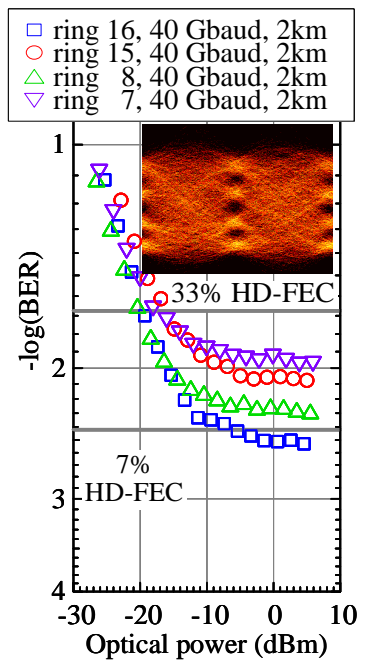

(c)

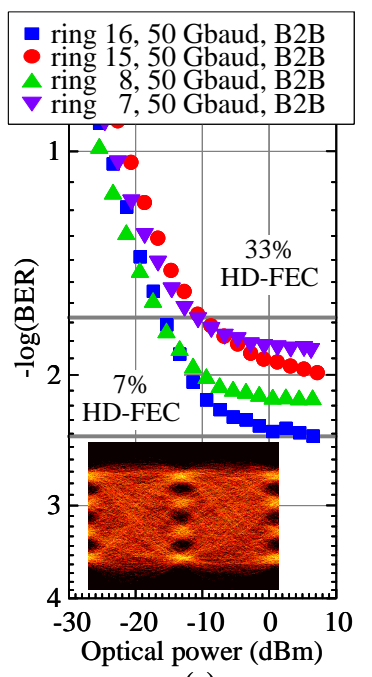

(e)

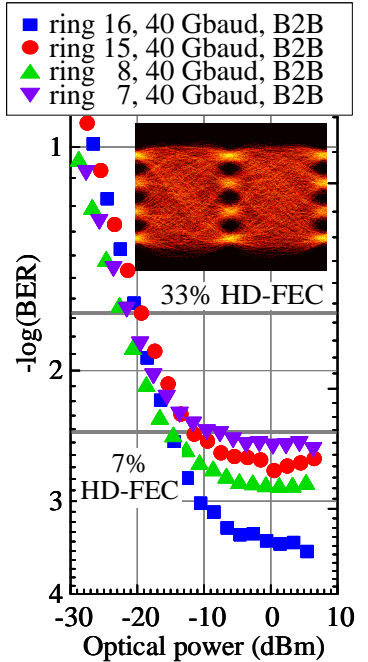

(b)

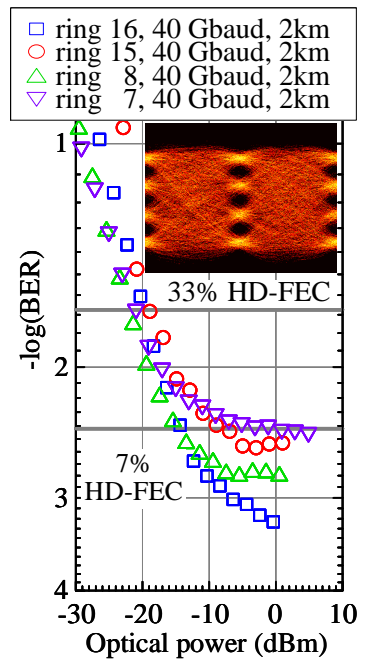

(d)

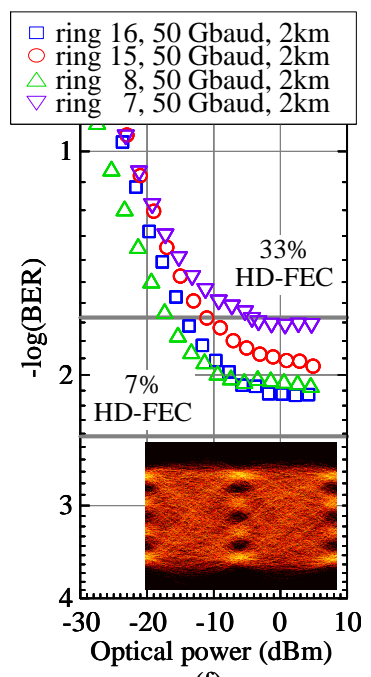

(f)
Fig. 6. BER performance of the $4 \times 40$ Gbaud DWDM-PAM 4 signals in back-to-back scenario using (a) LMS linear equalizer and (b) Volterra FFE, and after 2-km transmission using (c) LMS linear equalizer and (d) Volterra FFE; And BER performance of the $4 \times 50$ Gbaud DWDM-PAM 4 signals in (e) back-to-back scenario and (f) after 2-km transmission using Volterra FFE. 
modulation direct-detection based short-reach applications is not essential, we calculated the achieved SE for the DWDM system to be $1.04 \mathrm{bit} / \mathrm{s} / \mathrm{Hz}$. The SE can be improved by reducing the channel spacing and using higher-order PAM modulation. However, the complexity of channel filtering, intermodulation crosstalk [40], and decoding should be considered.

\section{CONCLUSION}

We have proposed an intra-datacenter interconnect scheme using the OFCM with an E/O frequency comb. Taking advantage of the strong wavelength-selectivity of the MRMs, the OFCM works as a frequency comb modulator without the need of DWDM multiplexer or demultiplexer. We have demonstrated $300 \mathrm{~Gb} / \mathrm{s}$ net-rate intra-datacenter interconnects using the OFCM. $4 \times 40$ Gbaud and $4 \times 50$ Gbaud DWDMPAM4 signals have been generated and transmitted through a 2-km SMF, with BER performance below $7 \%$ and $33 \%$ HD-FEC thresholds, respectively. The resulting net rates for the 40 Gbaud and 50 Gbaud DWDM signals are 299.06 $\mathrm{Gb} / \mathrm{s}$ and $300.75 \mathrm{~Gb} / \mathrm{s}$. The bandwidth of the MRMs is the main limiting factor for the achievable data rate. Given the small footprint of the OFCM and the broad bandwidth of the optical frequency comb, this scheme has the potential for a high capacity integrated transmitter for intra-datacenter interconnects with high density and low power consumption.

\section{REFERENCES}

[1] J. Witzens, "High-Speed Silicon Photonics Modulators," Proceedings of the IEEE, vol. 106, no. 12, pp. 2158-2182, dec 2018. [Online]. Available: https://ieeexplore.ieee.org/document/8540485/

[2] W. Bogaerts, S. K. Selvaraja, P. Dumon, J. Brouckaert, K. De Vos, D. Van Thourhout, and R. Baets, "Silicon-on-Insulator Spectral Filters Fabricated With CMOS Technology," IEEE Journal of Selected Topics in Quantum Electronics, vol. 16, no. 1, pp. 33-44, 2010.

[3] S. Pathak, M. Vanslembrouck, P. Dumon, D. Van Thourhout, and W. Bogaerts, "Optimized Silicon AWG With Flattened Spectral Response Using an MMI Aperture," Journal of Lightwave Technology, vol. 31, no. 1, pp. 87-93, 2013.

[4] M. Piels and J. E. Bowers, "1 - Photodetectors for silicon photonic integrated circuits," in Photodetectors, B. Nabet, Ed. Woodhead Publishing, 2016, pp. 3-20. [Online]. Available: http://www.sciencedirect.com/science/article/pii/B9781782424451000014

[5] J. Liu, D. Pan, S. Jongthammanurak, K. Wada, L. C. Kimerling, and J. Michel, "Design of monolithically integrated GeSi electroabsorption modulators and photodetectors on an SOI platform," Opt. Express, vol. 15, no. 2, pp. 623-628, 2007. [Online]. Available: http://www.opticsexpress.org/abstract.cfm?URI=oe-15-2-623

[6] S. A. Srinivasan, M. Pantouvaki, S. Gupta, H. T. Chen, P. Verheyen, G. Lepage, G. Roelkens, K. Saraswat, D. V. Thourhout, P. Absil, and J. V. Campenhout, " $56 \mathrm{~Gb} / \mathrm{s}$ Germanium Waveguide Electro-Absorption Modulator," Journal of Lightwave Technology, vol. 34, no. 2, pp. 419424, 2016

[7] P. D. Heyn, V. I. Kopp, S. A. Srinivasan, P. Verheyen, J. Park, M. S. Wlodawski, J. Singer, D. Neugroschl, B. Snyder, S. Balakrishnan, G. Lepage, M. Pantouvaki, P. Absil, and J. V. Campenhout, "Ultra-Dense $16 \times 56 \mathrm{~Gb} / \mathrm{s}$ NRZ GeSi EAM-PD Arrays Coupled to Multicore Fiber for Short-Reach 896Gb/s Optical Links," in Optical Fiber Communication Conference. Optical Society of America, 2017, p. Th1B.7. [Online]. Available: http://www.osapublishing.org/abstract.cfm?URI=OFC-2017Th1B.7

[8] F. Zhang, Y. Zhu, F. Yang, L. Zhang, X. Ruan, Y. Li, and Z. Chen, "Up to single lane 200G optical interconnects with silicon photonic modulator," in Optical Fiber Communication Conference Postdeadline Papers 2019. Optical Society of America, 2019, p. Th4A.6. [Online]. Available: http://www.osapublishing.org/abstract.cfm?URI=OFC-2019-Th4A.6
[9] E. El-Fiky, A. Samani, D. Patel, M. Jacques, M. Sowailem, and D. V. Plant, " $400 \mathrm{~Gb} / \mathrm{s}$ O-band silicon photonic transmitter for intra-datacenter optical interconnects," Optics Express, vol. 27, no. 7, p. 10258, 2019. [Online]. Available: https://www.osapublishing.org/abstract.cfm?URI=oe-27-7-10258

[10] A. Samani, D. Patel, M. Chagnon, E. El-Fiky, R. Li, M. Jacques, N. Abadía, V. Veerasubramanian, and D. V. Plant, "Experimental parametric study of $128 \mathrm{~Gb} / \mathrm{s}$ PAM-4 transmission system using a multi-electrode silicon photonic Mach Zehnder modulator," Opt. Express, vol. 25, no. 12, pp. 13 252-13 262, 2017. [Online]. Available: http://www.opticsexpress.org/abstract.cfm?URI=oe-25-12-13252

[11] P. Dong, C. Xie, L. Chen, L. L. Buhl, and Y.-K. Chen, "112$\mathrm{Gb} / \mathrm{s}$ monolithic PDM-QPSK modulator in silicon," Opt. Express, vol. 20, no. 26, pp. B624-B629, 2012. [Online]. Available: http://www.opticsexpress.org/abstract.cfm?URI=oe-20-26-B624

[12] P. Dong, J. Lee, Y.-K. Chen, L. L. Buhl, S. Chandrasekhar, J. H. Sinsky, and K. Kim, "Four-Channel 100-Gb/s Per Channel Discrete Multitone Modulation Using Silicon Photonic Integrated Circuits," Journal of Lightwave Technology, vol. 34, no. 1, pp. 79-84, 2016. [Online]. Available: http://ieeexplore.ieee.org/document/7360865/

[13] Q. Xu, B. Schmidt, S. Pradhan, and M. Lipson, "Micrometrescale silicon electro-optic modulator," Nature, vol. 435, no. 7040, pp. 325-327, 2005. [Online]. Available: https://doi.org/10.1038/nature03569

[14] J. Sun, R. Kumar, M. Sakib, J. B. Driscoll, H. Jayatilleka, and H. Rong, "A $128 \mathrm{~Gb} / \mathrm{s}$ PAM4 Silicon Microring Modulator With Integrated Thermo-Optic Resonance Tuning," Journal of Lightwave Technology, vol. 37, no. 1, pp. 110-115, 2019.

[15] P. Dong, R. Shafiiha, S. Liao, H. Liang, N.-N. Feng, D. Feng, G. Li, X. Zheng, A. V. Krishnamoorthy, and M. Asghari, "Wavelength-tunable silicon microring modulator," Opt. Express, vol. 18, no. 11, pp. 10941-10946, 2010. [Online]. Available: http://www.opticsexpress.org/abstract.cfm?URI=oe-18-11-10941

[16] P. Dong, J. Lee, K. Kim, Y.-K. Chen, and C. Gui, "TenChannel Discrete Multi-Tone Modulation Using Silicon Microring Modulator Array," in Optical Fiber Communication Conference. Optical Society of America, 2016, p. W4J.4. [Online]. Available: http://www.osapublishing.org/abstract.cfm?URI=OFC-2016-W4J.4

[17] Y. Zhu, F. Zhang, F. Yang, L. Zhang, X. Ruan, Y. Li, and Z. Chen, "Toward Single Lane 200G Optical Interconnects With Silicon Photonic Modulator," Journal of Lightwave Technology, vol. 38, no. 1, pp. 67-74, 2020.

[18] A. N. Pilipetskii, A. V. Turukhin, C. R. Davidson, D. G. Foursa, H. G. Batshon, J.-X. Cai, M. Mazurczyk, M. A. Bolshtyansky, O. V. Sinkin, and Y. Sun, "High Capacity Ultralong-Haul Power Efficient Transmission Using 12-Core Fiber," Journal of Lightwave Technology, vol. 35, no. 4, pp. 1028-1032, 2017. [Online]. Available: https://www.osapublishing.org/jlt/abstract.cfm?uri=jlt-35-4-1028

[19] E. El-Fiky, M. Osman, A. Samani, C. Gamache, M. H. Ayliffe, J. Li, M. Jacques, Y. Wang, A. Kumar, and D. V. Plant, "First demonstration of a $400 \mathrm{gb} / \mathrm{s} 4 \lambda \mathrm{cwdm}$ tosa for datacenter optical interconnects," Opt. Express, vol. 26, no. 16, pp. 19742-19749, 2018. [Online]. Available: http://www.opticsexpress.org/abstract.cfm?URI=oe-26-16-19742

[20] R. Dubé-Demers, S. LaRochelle, and W. Shi, "Ultrafast pulseamplitude modulation with a femtojoule silicon photonic modulator," Optica, vol. 3, no. 6, pp. 622-627, 2016. [Online]. Available: http://www.osapublishing.org/optica/abstract.cfm?URI=optica-3-6-622

[21] M. Zhang, B. Buscaino, C. Wang, A. Shams-Ansari, C. Reimer, R. Zhu, J. M. Kahn, and M. Lončar, "Broadband electrooptic frequency comb generation in a lithium niobate microring resonator," Nature, vol. 568, no. 7752, pp. 373-377, 2019. [Online]. Available: http://dx.doi.org/10.1038/s41586-019-1008-7 http://www.nature.com/articles/s41586-019-1008-7

[22] M. H. Khan, H. Shen, Y. Xuan, L. Zhao, S. Xiao, D. E. Leaird, A. M. Weiner, and M. Qi, "Ultrabroad-bandwidth arbitrary radiofrequency waveform generation with a silicon photonic chip-based spectral shaper," Nature Photonics, vol. 4, no. 2, pp. 117-122, 2010. [Online]. Available: https://doi.org/10.1038/nphoton.2009.266

[23] Sai Tak Chu, B. E. Little, Wugen Pan, T. Kaneko, and Y. Kokubun, "Cascaded microring resonators for crosstalk reduction and spectrum cleanup in add-drop filters," IEEE Photonics Technology Letters, vol. 11, no. 11 , pp. 1423-1425, 1999.

[24] D. Nikolova, D. M. Calhoun, Y. Liu, S. Rumley, A. Novack, T. Baehr-Jones, M. Hochberg, and K. Bergman, "Modular architecture for fully non-blocking silicon photonic switch fabric," Microsystems \& Nanoengineering, vol. 3, no. 1, p. 16071, 2017. [Online]. Available: https://doi.org/10.1038/micronano.2016.71 
[25] J. Dong, A. Zheng, D. Gao, S. Liao, L. Lei, D. Huang, and X. Zhang, "High-order photonic differentiator employing on-chip cascaded microring resonators," Opt. Lett., vol. 38, no. 5, pp. 628-630, 2013. [Online]. Available: http://ol.osa.org/abstract.cfm?URI=ol-38-5-628

[26] D. Brunina, X. Zhu, K. Padmaraju, L. Chen, M. Lipson, and K. Bergman, "10-Gb/s WDM Optically-Connected Memory System using Silicon Microring Modulators," in European Conference and Exhibition on Optical Communication. Optical Society of America, 2012, p. Mo.2.A.5. [Online]. Available: http://www.osapublishing.org/abstract.cfm?URI=ECEOC-2012Mo.2.A.5

[27] J. Flueckiger, S. M. Grist, G. Bisra, L. Chrostowski, and K. C. Cheung, "Cascaded silicon-on-insulator microring resonators for the detection of biomolecules in PDMS microfluidic channels," in Microfluidics, BioMEMS, and Medical Microsystems IX, H. Becker and B. L. Gray, Eds., vol. 7929, International Society for Optics and Photonics. SPIE, 2011, pp. 125-134. [Online]. Available: https://doi.org/10.1117/12.873974

[28] Q. Xu, B. Schmidt, J. Shakya, and M. Lipson, "Cascaded silicon micro-ring modulators for WDM optical interconnection," Opt. Express, vol. 14, no. 20, pp. 9431-9436, 2006. [Online]. Available: http://www.opticsexpress.org/abstract.cfm?URI=oe-14-20-9431

[29] C.-H. Chen, M. Ashkan Seyedi, M. Fiorentino, D. Livshits, A. Gubenko, S. Mikhrin, V. Mikhrin, and R. G. Beausoleil, "A comb laser-driven DWDM silicon photonic transmitter based on microring modulators," Optics Express, vol. 23, no. 16, pp. 21 541-21 548, 2015. [Online]. Available: http://www.opticsexpress.org/abstract.cfm?URI=oe23-16-21541

[30] M. A. Seyedi, C.-H. Chen, M. Fiorentino, and R. Beausoleil, "Error-free DWDM transmission and crosstalk analysis for a silicon photonics transmitter," Opt. Express, vol. 23, no. 26, pp. 32968-32976, 2015. [Online]. Available: http://www.opticsexpress.org/abstract.cfm?URI=oe23-26-32968

[31] W. M. J. Green, M. J. Rooks, L. Sekaric, and Y. A. Vlasov, "Ultracompact, low RF power, $10 \mathrm{~Gb} / \mathrm{s}$ silicon Mach-Zehnder modulator," Opt. Express, vol. 15, no. 25, pp. 17 106-17 113, 2007. [Online]. Available: http://www.opticsexpress.org/abstract.cfm?URI=oe-15-25-17106

[32] P. Dong, R. Gatdula, K. Kim, J. H. Sinsky, A. Melikyan, Y.-K. Chen, G. de Valicourt, and J. Lee, "Simultaneous wavelength locking of microring modulator array with a single monitoring signal," Opt. Express, vol. 25, no. 14, pp. 16040-16046, 2017. [Online]. Available: http://www.opticsexpress.org/abstract.cfm?URI=oe-25-14-16040

[33] R. Gatdula, K. Kim, A. Melikyan, Y.-K. Chen, and P. Dong, "Simultaneous four-channel thermal adaptation of polarization insensitive silicon photonics WDM receiver," Opt. Express, vol. 25, no. 22, pp. $27119-27126$, 2017. [Online]. Available: http://www.opticsexpress.org/abstract.cfm?URI=oe-25-22-27119

[34] M. A. Soto, M. Alem, M. Amin Shoaie, A. Vedadi, C.-S. Brès, L. Thévenaz, and T. Schneider, "Optical sinc-shaped Nyquist pulses of exceptional quality," Nature Communications, vol. 4, p. 2898, 2013. [Online]. Available: https://doi.org/10.1038/ncomms3898 http://10.0.4.14/ncomms3898

[35] J. Wu, J. Zang, Y. Li, D. Kong, J. Qiu, S. Zhou, J. Shi, and J. Lin, "Investigation on Nyquist pulse generation using a single dual-parallel Mach-Zehnder modulator," Opt. Express, vol. 22, no. 17, pp. 20463-20472, 2014. [Online]. Available: http://www.opticsexpress.org/abstract.cfm?URI=oe-22-17-20463

[36] Q. Wang, L. Huo, Y. Xing, and B. Zhou, "Ultra-flat optical frequency comb generator using a single-driven dual-parallel Mach-Zehnder modulator," Opt. Lett., vol. 39, no. 10, pp. 3050-3053, 2014. [Online]. Available: http://ol.osa.org/abstract.cfm?URI=ol-39-10-3050

[37] M. He, M. Xu, Y. Ren, J. Jian, Z. Ruan, Y. Xu, S. Gao, S. Sun, X. Wen, L. Zhou, L. Liu, C. Guo, H. Chen, S. Yu, L. Liu, and X. Cai, "High-performance hybrid silicon and lithium niobate MachZehnder modulators for $100 \mathrm{Gbit} / \mathrm{s}$ and beyond," Nature Photonics, vol. 13, no. 5, pp. 359-364, 2019. [Online]. Available: https://doi.org/10.1038/s41566-019-0378-6

[38] N. Stojanovic, F. Karinou, Z. Qiang, and C. Prodaniuc, "Volterra and Wiener Equalizers for Short-Reach 100G PAM-4 Applications," Journal of Lightwave Technology, vol. 35, no. 21, pp. 4583-4594, 2017.

[39] K. Zhang, Q. Zhuge, H. Xin, W. Hu, and D. V. Plant, "Performance comparison of DML, EML and MZM in dispersion-unmanaged short reach transmissions with digital signal processing," Opt. Express, vol. 26, no. 26, pp. 34288-34 304, 2018. [Online]. Available: http://www.opticsexpress.org/abstract.cfm?URI=oe-26-26-34288

[40] K. Padmaraju, X. Zhu, L. Chen, M. Lipson, and K. Bergman, "Intermodulation Crosstalk Characteristics of WDM Silicon
Microring Modulators," IEEE Photonics Technology Letters, vol. 26, no. 14, pp. 1478-1481, jul 2014. [Online]. Available: http://ieeexplore.iee.org/document/6824827/

[41] L. M. Zhang and F. R. Kschischang, "Staircase Codes With 6\% to 33\% Overhead," J. Lightwave Technol., vol. 32, no. 10, pp. 1999-2002, 2014. [Online]. Available: http://jlt.osa.org/abstract.cfm?URI=jlt-32-10-1999 